\title{
Nivel de pensamiento crítico de estudiantes de primero y último semestre de pregrado en enfermería de una universidad en Medellín, Colombia
}

\author{
Critical thinking level of undergraduate nursing students from first and last semesters in a university of Medellin, Colombia
}

Diego Alejandro Salazar-Blandón ${ }^{*}$ orcid.org/0000-0002-8724-7705

Beatriz Elena Ospina-Rave' orcid.org/0000-0003-0446-4649

1 Universidad de Antioquia. Medellín, Colombia

\begin{abstract}
Resumen
Introducción: El pensamiento crítico es un componente importante de la formación en Enfermería. Diferentes organizaciones exigen estrategias de pensamiento crítico para acreditar programas universitarios. Sin embargo, existe poca evidencia sobre la medición de sus niveles. Objetivo: Comparar el nivel de pensamiento crítico, en estudiantes de primero y último semestre de Enfermería en una universidad de Medellín-Colombia. Materiales y métodos: Estudio cuantitativo descriptivo, trasversal y comparativo del pensamiento crítico en una muestra a conveniencia de estudiantes de primero y último semestre. Resultados: En ambos grupos, el nivel es medio (primero 44,47; último 53,14 p<0,001). No obstante, se observa un incremento de 19,49\% con respecto al primer semestre, con cambios significativos en los dominios: evaluación, argumentación e interpretación. No se encontraron diferencias en los dominios de inferencia y análisis. Conclusiones: Los hallazgos muestran un cambio positivo en el nivel de pensamiento crítico, que son un punto de partida para el análisis del proceso enseñanza aprendizaje en la facultad donde se desarrolló el estudio, con el fin de fortalecer los dominios donde se observaron incrementos significativos y desarrollar estrategias que favorezcan el desarrollo de habilidades en los que no se observaron cambios. Es importante desarrollar investigaciones con mediciones longitudinales e instrumentos confiables.
\end{abstract}

Palabras clave: Pensamiento; enfermería; educación superior. (Fuente: DeCS, Bireme).

\begin{abstract}
Introduction: Critical thinking is an important component of nursing education. Different organizations demand critical thinking strategies in order to accredit university programs. Nevertheless, there is limited evidence in regards to how critical thinking levels can be assessed. Objective: To compare the critical thinking levels of undergraduate nursing students from first and last semesters in a university of Medellin-Colombia. Materials and methods: A quantitative, descriptive, crosssectional and comparative study of critical thinking carried out with an appropriate sample of students from first and last semesters. Results: Both groups showed a medium level (44.47 and 53.14 for first and last semester students, respectively). However, there is a $19.49 \%$ increase in last semester students compared to ones belonging to first semester, with significant changes in domains like evaluation, argumentation and interpretation. We did not observe significant changes in inference and analysis. Conclusions: Our findings show a positive change in the critical thinking level, a result that can be used as a starting point for the analysis of the teaching-learning processes developed in the Nursing Faculty. Our results are also important for strengthening the domains that showed significant increments and developing strategies to foster the development of skills in the areas where changes were not evident. We consider that it is relevant to carry out studies with longitudinal measurements and reliable instruments.
\end{abstract}

Key words: Thinking; nursing; education, higher. (Source: DeCS, Bireme).

\footnotetext{
*Autor de correspondencia

Diego Alejandro Salazar Blandón

e-mail: alejandro.salazar@udea.edu.co
} 


\section{Introducción}

El pensamiento crítico (PC) es un concepto relevante en la educación, más aún en la educación superior, ya que es una herramienta útil para que el estudiante aprenda a filtrar la información, a desaprender y a transformarse. En un entorno dinámico impulsado por las tecnologías de la información y la comunicación, los futuros profesionales se enfrentarán a cambios, ambigüedades e información contradictoria, que sin duda los obligará a tomar decisiones difíciles y a solucionar problemas complejos en su desempeño profesional. Por ello, las habilidades cognitivas de orden superior, como el PC, han ganado gradualmente importancia en la educación superior(1); pensar críticamente es más importante que tener buenas calificaciones o buenos promedios académicos, aunque lo primero pueda influenciar, o no, lo segundo; por otro lado, el promedio académico tiene un uso limitado en el desempeño profesional(2).

La enfermería se distingue como una disciplina con una elevada diversidad y complejidad respecto a su práctica y labor cotidiana. A lo anterior, se suma los avances tecnológicos que han logrado que el paciente pase de ser un receptor pasivo, a una persona que cuestiona y toma decisiones sobre su proceso de atención y cuidado(3). Así mismo, los cambios políticos y sociales de las últimas décadas han trasformado las organizaciones de salud, reclamando una mejor calidad de los servicios a un menor costo. Este contexto hace que día a día, se presenten diversas situaciones en torno al cuidado, que le plantean al profesional de enfermería, la necesidad de buscar alternativas, desde diferentes enfoques para la toma de decisiones, con un sentido crítico. La Enfermería Basada en la Evidencia (EBE) ${ }^{(4)}$ y la formación basada en competencias (FBC), precisan una visión compresiva del escenario actual, para propiciar una mejor adaptación al contexto social, lo que hace que estos enfoques sean esenciales y prioritarios en ciencias de la salud(5), para formar desde los conocimientos, habilidades y actitudes en enfermería.

Cárdenas, et al.(6), afirman que en la actualidad, en enfermería se distinguen más las habilidades cognitivas que las psicomotoras que históricamente habían sido sobredimensionadas en la formación del profesional. Entre las habilidades cognitivas sobresale el PC, a tal punto que diferentes organizaciones y universidades a nivel mundial, se han propuesto incluir en sus currículos de formación profesional en enfermería, estrategias que promuevan su desarrollo. Es el caso de Asociación Americana de Colegios de Enfermería $(\mathrm{AACN})^{(7)}$ y la Liga Nacional de Enfermería (NLN) ${ }^{(8)}$, en Estados Unidos, que desde 1989 reconoce la inclusión de competencias en PC como requisito para la acreditación de programas universitarios, o como se evidencia en los esfuerzos hechos por la Red Iberoamericana de Investigación en Educación en Enfermería (RIIEE), por indagar y resaltar la importancia del PC en la educación en enfermería en la región $(6,9,10)$.

No obstante, reconocer la importancia del PC en la formación del profesional en Enfermería, e incluir en los perfiles de formación y en los currículos competencias en esta dirección, no garantiza que se logre formar pensadores críticos, como lo demuestran en su investigación Cárdenas, et al.(10), al concluir que para el ámbito iberoamericano, el PC en enfermería es un proceso complejo que tiene varios sentidos, con base en el enfoque teórico o en el contexto del que se parta.

Si bien han existido esfuerzos por tener un consenso sobre la definición de PC, como el realizado por la Sociedad Americana de Filosofía (APA)(11), siguen presentándose definiciones posteriores, como lo evidencian Basco, et al.(12), cuando presentan, en su revisión 10 visiones de diferentes autores en un periodo comprendido entre 2001 y 2013. Pese a lo complejo del constructo, en la actualidad se dispone de diferentes instrumentos para su medición, tales como el California Critical Thinking, Critical Thinking, Appraisal, Halpern Critical Thinking, Health Sciences Reasoning, PENCRISAL o el Critical Thinking Diagnostic. Para esta investigación, se optó por una versión del instrumento propuesto por Ospina, et al.(13), en 2016, desarrollado en Medellín-Colombia, validado con estudiantes de enfermería y sociología, y mide el nivel de PC mediante las habilidades de evaluación, argumentación, análisis, inferencia e interpretación.

En Iberoamérica, son escasas las investigaciones cuantitativas sobre la medición de los niveles de PC en la formación del profesional de enfermería(14), por lo que resulta imperativo establecer puntos de partida que permitan identificar los niveles actuales de PC al inicio y al final de la formación profesional, para identificar cuáles son los cambios que se 
presentan en las diferentes habilidades que se miden con el instrumento, y proponer estrategias que permitan fortalecer, o mejorar, dichos niveles en el tiempo, y así lograr que la formación universitaria de esta disciplina pueda desarrollar esta importante habilidad cognitiva al futuro profesional.

En este sentido, la investigación busca comparar los niveles de PC en los estudiantes de enfermería al inicio y al final de su carrera, teniendo en cuenta los dominios de evaluación, argumentación, análisis, inferencia e interpretación.

\section{Materiales y métodos}

Se realizó un estudio de tipo cuantitativo, descriptivo, transversal y comparativo, con una población de estudiantes de pregrado en enfermería, matriculados en el semestre académico 2016/2 en una universidad en Medellín-Colombia. Todos los estudiantes de primero y último semestre que participaron de manera voluntaria en el estudio, fueron incluidos en el estudio por medio de un censo. Se excluyó del análisis a los estudiantes de intercambio o de movilidad académica, y a estudiantes que por llegar tarde a clase, o no tener una buena disposición hacia la investigación, no respondieron a más del $75 \%$ del cuestionario, y se retiraron de la prueba, obteniendo así una muestra a conveniencia de 104 participantes, de los 120 estudiantes matriculados.

Previo a la recolección de la información, se informó a los participantes los objetivos y beneficios de la investigación, y luego de firmar el consentimiento informado, se aplicó un cuestionario que incluye datos sociodemográficos y 50 ítems de selección múltiple, con única respuesta, para medir PC, cuyos resultados tienen un valor mínimo de 0 y máximo de 100. Este instrumento es una versión modificada del propuesto y vaidado por Ospina, et al.(13). En ambos instrumentos se calcula el índice global de PC, por medio de cinco subescalas de los dominios o dimensiones: argumentación, análisis, interpretación, inferencia y evaluación.

Para la confiabilidad y validez del instrumento utilizado en este estudio, se aplicó una prueba piloto en 90 estudiantes (55 de enfermería y 35 de sociología), matriculados en los primeros y últimos semestres de cada programa académico. Se obtuvo una confiabilidad por medio de alfa de Cronbach de 0,519 . Al aplicar el test en la población de estudio, se obtuvo un alfa de Cronbach de 0,659. Para la validez, se usó un análisis factorial confirmatorio, el cual reportó un índice KMO 0,52, y la prueba de Bartlett, con un $\mathrm{p}<0,001$. Se obtuvo una variabilidad del $69,48 \%$, es decir, los ítems construidos explican cerca del $70 \%$ de las dimensiones que conforman el PC, y además se encontró una alta concordancia entre la correlación del ítem y la dimensión a la cual pertenece, según la propuesta teórica.

Para el procesamiento de los datos se utilizó el software estadístico SPSS 23; se calculó medidas de tendencia central, dispersión y frecuencia de las variables sociodemográficas, del índice de PC y sus dimensiones. Se aplicó pruebas t-student para grupos independientes, con el fin de comparar las medias obtenidas en la valoración global de PC, y por dimensiones, entre el primero y el último semestre. Se usó el coeficiente de correlación de Spearman y pruebas chi-cuadrado para examinar posibles relaciones entre los niveles de PC y semestre académico de los estudiantes, según variables sociodemográficas. Se consideró, en todos los casos, un nivel de significancia estadística <0,05.

\section{Consideraciones éticas}

La investigación siguió los principios y normas éticas establecidas en la Resolución 8430 de 1993 del Ministerio de Salud colombiano, se garantizó la participación voluntaria y la confidencialidad de los estudiantes y fue aprobada por el Comité de Ética en Investigación de la Facultad de Enfermería de la Universidad de Antioquia.

\section{Resultados}

La población de estudio estuvo constituida por 55 estudiantes del primer semestre, y 49 del octavo, del pregrado en Enfermería. La edad promedio en el primer semestre fue de 19 años ( \pm 2 años), con un mínimo de 17 años y un máximo de 26 años, y en el último semestre fue de 23 años ( \pm 2 años), con un mínimo de 21 años y un máximo de 32 años. Se observó que 3 de cada 4 estudiantes (78\%) eran mujeres. El 60,6\% vive actualmente en Medellín, el $33 \%$ en otro municipio del área metropolitana, y el $5,8 \%$ restante en otros municipios del departamento de Antioquia. El 94,2\% pertenece a los estratos 1, 2 o 3. La mayoría de los participantes $(95,2 \%)$ se auto identifica como mestizo, el $2.9 \%$ como indígena y el 1,9\% como afrodescendiente. El $94,2 \%$ son solteros, el $93,1 \%$ no tiene hijos y 1 de cada 4 estudiantes son 
trabajadores. El 84,6\% egresó de un colegio público. Las características sociodemográficas de los estudiantes de cada semestre se presentan en la Tabla
1. Sólo se observan diferencias estadísticamente significativas en el sexo y en la proporción de estudiantes trabajadores.

Tabla 1. Características sociodemográficas de estudiantes de primero y último semestre de enfermería Medellín-Colombia

\begin{tabular}{|c|c|c|c|c|c|c|}
\hline \multirow{3}{*}{ Variable } & \multicolumn{4}{|c|}{ Semestre académico } & \multirow{3}{*}{$\chi 2$} & \multirow{3}{*}{$p$} \\
\hline & \multicolumn{2}{|c|}{ Primero } & \multicolumn{2}{|c|}{ Último } & & \\
\hline & $\mathbf{n}$ & $\%$ & $\mathbf{n}$ & $\%$ & & \\
\hline \multicolumn{7}{|l|}{ Sexo } \\
\hline Masculino & 6 & 10,91 & 16 & 32,65 & 7,346 & 0,007 \\
\hline Femenino & 49 & 89,09 & 33 & 67,35 & & \\
\hline \multicolumn{7}{|l|}{ Estado civil } \\
\hline Soltero & 54 & 98,18 & 44 & 89,80 & 3,352 & $0,097^{*}$ \\
\hline Casado & 1 & 1,82 & 5 & 10,20 & & \\
\hline \multicolumn{7}{|l|}{ Número de hijos } \\
\hline 0 & 51 & 98,08 & 43 & 87,76 & 5,59 & 0,133 \\
\hline 1 & 0 & 0,00 & 4 & 8,16 & & \\
\hline 2 & 0 & 0,00 & 1 & 2,04 & & \\
\hline 3 & 1 & 1,92 & 1 & 2,04 & & \\
\hline \multicolumn{7}{|l|}{ Estrato socioeconómico } \\
\hline 1 & 7 & 12,73 & 3 & 6,12 & 4,94 & 0,293 \\
\hline 2 & 22 & 40,00 & 14 & 28,57 & & \\
\hline 3 & 23 & 41,82 & 29 & 59,18 & & \\
\hline 4 & 2 & 3,64 & 3 & 6,12 & & \\
\hline 5 & 1 & 1,82 & 0 & 0,00 & & \\
\hline \multicolumn{7}{|c|}{ Terminó sus estudios secundarios en un colegio } \\
\hline Público & 46 & 83,64 & 42 & 85,71 & 0,086 & 0,769 \\
\hline Privado & 9 & 16,36 & 7 & 14,29 & & \\
\hline \multicolumn{7}{|l|}{ Trabajo actual } \\
\hline 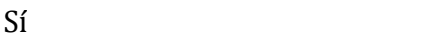 & 5 & 9,09 & 21 & 42,86 & 15,757 & $<0,001$ \\
\hline No & 50 & 90,91 & 28 & 57,14 & & \\
\hline
\end{tabular}

${ }^{*}$ Se aplicó prueba exacta de Fisher

No se encontró diferencias estadísticamente significativas entre los niveles de PC y el sexo. El nivel promedio de PC para hombres fue de 50,45 $\pm 13,20$, y para mujeres de 48,04 $\pm 9,79(\mathrm{t}=0,946, \mathrm{p}=0,346)$. El coeficiente de correlación de Pearson entre la edad y el nivel de PC fue de 0,322 ( $t=3,41 \mathrm{p}=0,01)$, donde se observó que existe una débil correlación entre los niveles de PC y la edad, lo que implica que, a medida que aumenta la edad, también lo hace el nivel de PC.

Los puntajes promedio en la valoración de PC y sus dominios se presentan en la Tabla 2. Los estudiantes del primer semestre obtuvieron mayor puntación promedio en los dominios análisis (capacidad de identificar las relaciones de inferencia, reales o supuestas, entre enunciados $u$ otras formas de representación, a partir de la evidencia disponible(15)) e interpretación (capacidad de comprender el significado de una amplia variedad de experiencias, situaciones y eventos. Implica la clasificación, la decodificación y la aclaración de un significado(15)). El puntaje más bajo se obtuvo en el dominio inferencia (identificar los elementos necesarios para derivar conjeturas e hipótesis, considerando la información disponible(15)). Respecto al índice global de PC, según Ospina, et al.(13), los estudiantes de primer semestre se ubican en un índice medio de PC.

Por su parte, los estudiantes de último semestre mostraron mejor desempeño respecto al primero, en los cinco dominios, obteniendo una mayor puntación en evaluación (valorar la credibilidad y fortaleza lógica en la información suministrada(15)), argumentación (justificar el razonamiento presentando los resultados con argumentos convincentes y evidenciables, considerando el contexto de la experiencia(15)), e interpretación, estos dominios presentaron diferencias estadísticamente 
significativas, y aunque el estudio fue de corte transversal, dichas diferencias no pueden ser atribuibles al azar (Tabla 2). No se determinó un cambio estadísticamente significativo entre los estudiantes de ambos semestres en relación a las habilidades de inferencia y análisis, manteniendo sus bajos niveles en la habilidad cognitiva inferencia, y con un cambio positivo en análisis. Se observó, además, un incremento de 8,67 puntos en la valoración general del índice de PC, pero, pese a esto, la puntación obtenida sigue ubicando a los estudiantes de último semestre en un nivel medio de PC.

Tabla 2. Comparación entre puntaje promedio obtenido y la valoración de PC y sus dominios

\begin{tabular}{|c|c|c|c|c|c|c|}
\hline \multirow{2}{*}{ Dominio } & \multicolumn{2}{|c|}{ Primero } & \multicolumn{2}{|c|}{ Último } & \multirow{2}{*}{$t$} & \multirow{2}{*}{$p$} \\
\hline & Media & D.E & Media & D.E & & \\
\hline Inferencia & 30 & 13,88 & 31,84 & 11,31 & 0,734 & 0,464 \\
\hline Evaluación & 40,36 & 22,77 & 54,08 & 16,7 & 3,467 & 0,001 \\
\hline Argumentación & 47,27 & 15,21 & 53,06 & 10,45 & 2,235 & 0,028 \\
\hline Análisis & 50,73 & 18,34 & 57,14 & 15,28 & 1,925 & 0,057 \\
\hline Interpretación & 54 & 22,82 & 69,59 & 19,79 & 3,701 & $<0,001$ \\
\hline Pensamiento crítico & 44,47 & 10,94 & 53,14 & 8,07 & 4,551 & $<0,001$ \\
\hline
\end{tabular}

\section{Discusión}

No se encontró diferencias estadísticamente significativas en los niveles promedios de PC al comparar por sexo, hallazgo similar a lo reportado por Salahshoor y Rafiee(16), quienes compararon los niveles de PC usando el instrumento de WatsonGlaser no encontraron diferencias entre hombres $(43,2 \pm 9,07)$ y mujeres $(40,94 \pm 7,37 \mathrm{p}=0,064)$ en una muestra de cinco institutos privados de idiomas en Irán. Existe una débil correlación directamente proporcional entre la edad y el nivel de PC, dado que los estilos de aprendizaje están influenciados por multitud de factores, entre ellos la edad, ya que se espera que, con el pasar de los años, se tenga un crecimiento personal e intelectual en los estudiantes(17) influenciado por el tipo de formación universitaria (18,19). Este resultado es contrario al reportado por Azizi, et al.(20), quienes encontraron una correlación inversamente proporcional ( $\mathrm{r}=-$ $0,172, p=0,06$ ), en una población de estudiantes de primero y último año de enfermería en Irán; sí mismo, Hunter, et al.(21), no encontraron diferencias estadísticamente significativas entre diferentes grupos de edad, y los niveles de PC, en estudiantes de enfermería en Australia.

Se observó que el nivel de PC de estudiantes de primer semestre es inferior al del último semestre (44,47 y 53,14 respectivamente). Sin embargo, el índice desarrollado por Ospina, et al.(13), ubica ambos valores, desde la construcción teórica, con un nivel de
PC medio, es importante resaltar que no se cuenta en el momento con un referente valorativo de PC con el cual comparar realmente los resultados que se obtienen de la medición del PC, como ocurre con el cociente intelectual IQ, que a menudo es comparado con el de personajes históricos como Albert Einstein(22).

No obstante, tener una medición objetiva del nivel PC en estudiantes que inician, y otra de estudiantes que finalizan su formación universitaria en enfermería, permite utilizar como referente la medición inicial, donde se observa una diferencia de 8,67 puntos en la escala de valoración de PC, lo cual equivale a un cambio de 19,49\%; estos resultados son similares a los reportados por Tahery, et al.(23), en 2008 en Irán, quienes, evaluaron el PC y sus dominios mediante un instrumento diferente (Watson-Glaser Critical Thinking) al usado en la presente investigación, pero reportaron niveles promedio de PC en tercer semestre de 42,29, y en último semestre de 49,0 $(\mathrm{p}<0,001), y$ también encontraron diferencias estadísticamente significativas en inferencia $(5,12$ $6,45 \mathrm{p}=0,010)$, reconocimiento de supuestos $(8,81$ $11,54 \mathrm{p}<0,001)$ e interpretación $(9,67-11,0 \mathrm{p}=0,003)$, resultados contrarios a los hallados por Azizi-Fini, et al.(20), quienes en 2015 midieron con el California Critical Thinking a estudiantes de primero y último semestre de un programa de enfermería en Irán, y no encontraron diferencias estadísticamente significativas en el nivel de PC y las habilidades por las que indaga el instrumento. 
Medir el PC, posibilita a las instituciones formadoras de profesionales en enfermería y a sus docentes, conocer el nivel de PC en sus estudiantes, e implementar estrategias intencionadas para el fortalecimiento o desarrollo de esta habilidad cognitiva y sus dominios. En este sentido, Carter, et al.(24), en su revisión, encontraron 28 estudios en 8 países, los cuales evaluaron 12 diferentes intervenciones de enseñanza para promover el PC en estudiantes de enfermería, entre las que se destacan, por sus buenos resultados, el aprendizaje basado en problemas, los mapas conceptuales, la simulación y la pedagogía narrativa; concluyen, que es necesario continuar diseñando estudios que permitan identificar las buenas prácticas pedagógicas para el fortalecimiento y desarrollo del PC.

Así mismo, revelan la importancia de mejorar los diseños metodológicos, con el fin de garantizar una medición objetiva de PC, la necesidad de un acertado control de posibles sesgos en la medición y una adecuada exposición a las estrategias educativas, que permitan mostrar cambios en los niveles de PC en el tiempo. Villatoro, et al.(25), exponen una propuesta metodológica de estrategias de enseñanza aprendizaje, con posibilidades comprobadas para potenciar de manera significativa el PC en estudiantes de enfermería, con aprendizaje basado en problemas y aprendizaje basado en retos. Por su parte, Carreño y Mayorga(26) afirman que la claridad sobre los conceptos estadísticos facilita el desarrollo del PC para ser mejores consumidores de literatura científica, lo que hace más efectiva la EBE.

Identificar las mejores estrategias para estimular el PC mediante la medición, proporciona herramientas para la inclusión del PC en el currículo de enfermería(27). Una vez incluidas estas estrategias, es posible valorar su impacto mediante la medición de PC, lo que permite direccionar esfuerzos o enfocar trasformaciones en este sentido, con lo cual se pueda garantizar que los programas de formación en enfermería promuevan efectivamente habilidades de PC.

Es una limitante de la presente investigación su enfoque descriptivo, que no permite establecer relaciones de causalidad o de correlación entre los factores y estrategias particulares que caracterizan la formación de los estudiantes de la universidad donde se llevó a cabo el estudio. Es importante considerar estudios posteriores con diseños analíticos y longitudinales, que permitan determinar el efecto en el tiempo de fomentar la formación de PC en enfermería.

\section{Conclusiones}

Los hallazgos muestran un cambio positivo en el nivel de pensamiento crítico en enfermería, que se convierte en un punto de partida para el análisis del proceso enseñanza aprendizaje en la facultad donde se desarrolló el estudio, con el fin de fortalecer los dominios en los que se observan incrementos estadísticamente significativos, y desarrollar estrategias que favorezcan el desarrollo de habilidades en los dominios en donde no se observaron cambios.

Ya está bien documentada la importancia del PC en la formación en Enfermería, por lo que es necesario avanzar a la acción, implementando estrategias que vayan más allá de la inclusión de competencias en los currículos o perfiles de formación. Se deben proponer pedagogías didácticas y políticas institucionales intencionadas hacia el fomento del PC, acompañadas de mediciones objetivas de los niveles PC y sus dominios, con instrumentos validados y confiables que evidencien el logro del objetivo.

Artículo ligado a investigación: "Nivel de desarrollo del pensamiento crítico en estudiantes de primero y último nivel académico de las áreas de ciencias sociales y salud, al ingreso y durante su formación profesional universitaria" Código: Enf012015.

\section{Financiación}

Comité para el Desarrollo de la Investigación CODI Universidad de Antioquia.

\section{Conflictos de interés: Ninguno.}

\section{Referencias}

1. Franco A, Almeida L, Saiz C. Pensamiento crítico: reflexión sobre su lugar en la enseñanza superior. Educ Siglo XXI [Internet]. 2014;32(2):81-96. Available from: https://revistas.um.es/educatio/article/view/202171/164731

2. Facione P. Critical thinking: What it is and why it counts [Internet]. Millbrae: The California Academic Press; 2011. Available from: http://www.student.uwa.edu.au/_data/assets/pdf_file/0003/ 1922502/Critical-Thinking-What-it-is-and-why-it-counts.pdf

3. Olmos C. Los medios de comunicación y la atención médica. Rev CONAMED [Internet]. 2010;15(1):37-42. Available from: http://www.dgdi-conamed.salud.gob.mx/ojsconamed/index.php/revconamed/article/view/269/490 
4. Eterovic C, Stiepovich J. Enfermería basada en la evidencia y formación profesional. Cienc y Enfermería [Internet]. 2010;16(3):9-14. Available from: https://scielo.conicyt.cl/pdf/cienf/v16n3/art_02.pdf

5. Millán J, Palés J, Morán J. Principios de educación médica: desde el grado hasta el desarrollo profesional. España: Editorial Médica Panamericana SA; 2015.

6. Cárdenas L, Dolores M, Porras B, Antonia M, Gómez J, De VA. Presencia del pensamiento reflexivo y crítico en los currículos de enfermería en Iberoamérica: una visión cualitativa [Internet]. Investigación Cualitativa en Educación. 2016;1:128-37. Available

from: https://proceedings.ciaiq.org/index.php/ciaiq2016/article/do wnload/596/585/

7. American Association of Colleges of Nursing. Fact Sheet: The impact of education on nursing practice [Internet]. Washington: AACN; 2014.2 Available from: https://www.aacnnursing.org/Portals/42/News/Factsheets/E dImpact.pdf

8. National League for Nursing - Commission for Nursing Education Accreditation. Accreditation standards for nursing education programs [Internet]. Washington: NLN-CNEA; 2016. Available from: http://www.nln.org/docs/default-source/accreditationservices/cnea-standards-final-february-

201613f2bf5c78366c709642ff00005f0421.pdf

9. Cárdenas L, Monroy A, Arana B, García M. Importancia del pensamiento reflexivo y crítico en enfermería. Rev Mex Enfermería Cardiológica [Internet]. 2015;23(1):35-41. Available from: http://www.medigraphic.com/pdfs/enfe/en2015/en151f.pdf

10. Cárdenas L, Bardallo $M$, Jiménez $M$, Lenise $M$, Martínez B. Desarrollo del pensamiento reflexivo y crítico en los estudiantes de enfermería de Iberoamérica. Estado del arte [Internet]. Investigación Cualitativa en Educación. 2015;2:400-4. Available from:

https://proceedings.ciaiq.org/index.php/ciaiq2015/article/vie $\mathrm{w} / 284 / 280$

11. Facione P. Critical Thinking: A statement of expert consensus for purposes of educational assessment and instruction. Executive summary: "The Delphi Report" [Internet]. Millbrae: California Academic Press; 1990;1-18. Available from: http://www.qcc.cuny.edu/SocialSciences/ppecorino/CTExpert-Report.pdf

12. Basco L, Rodríguez N, Puig M, Lluch T, Giménez M, Fariñas S. Revisión del concepto de Pensamiento Crítico en Enfermería: definición, composición, contextualización y medición. NURE Investig [Internet]. 2016;13(83):1-13. Available from: http://www.nureinvestigacion.es/0JS/index.php/nure/article/ view/774/717

13. Ospina B, Brand E, Aristizábal C. Development of a measurement index of critical thinking in professional formation. Investig y Educ en Enfermería [Internet]. 2017;35(1):69-77. Available from: http://www.scielo.org.co/pdf/iee/v35n1/2216-0280iee-35-01-00069.pdf

14. Zuriguel E, Lluch M, Falcó A, Puig M, Moreno C, Roldán J. Critical thinking in nursing: Scoping review. Int J Nurs Pract [Internet]. 2015;21(6):820-30. Available from: https://onlinelibrary.wiley.com/doi/full/10.1111/ijn.12347

15. Salazar DA, Ospina BE. Nivel de desarrollo del pensamiento crítico en estudiantes de primero y último nivel académico de las áreas de ciencias sociales y salud, al ingreso y durante su formación profesional universitaria. [Informe científico] Universidad de Antioquia; 2017.
16. Salahshoor N, Rafiee M. The relationship between Critical Thinking and gender: A case of Iranian EFL learners. J Appl Linguist Lang Res [Internet]. 2016;3(2):117-23. Available from: http://www.jallr.com/index.php/JALLR/article/viewFile/219/ pdf 219

17. Monroy F, Hernández F. Factores que influyen en los enfoques de aprendizaje universitario. Una revisión sistemática. Educ XXI [Internet]. 2014;17(2):105-24. Available from: http://revistas.uned.es/index.php/educacionXX1/article/view /11481/11437

18. Matalinares M. Relación entre el estilo de aprendizaje y la profesión elegida por estudiantes universitarios. Rev Investig en Psicol [Internet]. 2003;6(1):73-87. Available from: http://revistasinvestigacion.unmsm.edu.pe/index.php/psico/a rticle/view/5091/5378

19. Freiberg A, Fernández M. Estilos de aprendizaje en estudiantes universitarios ingresantes y avanzado de Buenos Aires. Liberabit [Internet]. 2015;21(1):71-9. Available from: http://www.scielo.org.pe/pdf/liber/v21n1/a07v21n1.pdf

20. Azizi-Fini I, Hajibagheri A, Adib-Hajbaghery M. Critical thinking skills in nursing students: A comparison between freshmen and senior students. Nurs Midwifery Stud [Internet]. 2015;4(1):1-5. Available from: https://www.ncbi.nlm.nih.gov/pmc/articles/PMC4377532/

21. Hunter S, Pitt V, Croce N, Roche J. Critical thinking skills of undergraduate nursing students: Description and demographic predictors. Nurse Educ Today [Internet]. 2014;34(5):809-14. Available from: https://www.sciencedirect.com/science/article/pii/S0260691 713002918?via\%3Dihub

22. Cox C. Genetic studies of genius: The early mental traits of three hundred geniuses. Vol. 2. Stanford: Stanford University Press; 1926. $842 \mathrm{p}$.

23. Tahery N, Cheraghian B, Esmaiili T, Mohammadi H, Aboodi J. Comparison of critical thinking skills between continuous licentiate and bachelor- licentiate nursing students of Abadan nursing faculty in 2008. Educ Res Med Sci [Internet]. 2012;1(1):1-5. Available from: https://cdn.neoscriber.org/cdn/dl/cb7a4bfa-51c4-11e8-83a3a32aecd579ec

24. Carter A, Creedy D, Sidebotham M. Efficacy of teaching methods used to develop critical thinking in nursing and midwifery undergraduate students: A systematic review of the literature. Nurse Educ Today [Internet]. 2016;40:209-18. Available from: https://www.sciencedirect.com/science/article/pii/S0260691 716001167

25. Villatoro J, Pérez E. El pensamiento crítico en la formación de estudiantes de enfermería: propuestas metodológicas para profesores. Salud en Chiapas [Internet]. 2017;5(3):130-6. Available from: http://revista.saludchiapas.gob.mx/api/v1/recursos/articulos/ 195

26. Carreño S, Mayorga J. Pensamiento estadístico: herramienta para el desarrollo de la enfermería como ciencia. Av en Enfermería [Internet]. 2017;35(3):345-56. Available from: http://www.scielo.org.co/pdf/aven/v35n3/0121-4500-aven35-03-00345.pdf

27. Burrell A. Integrating critical thinking strategies into nursing curricula. Teach Learn Nurs [Internet]. 2014;9(2):53-8. Available from: https://www.sciencedirect.com/science/article/pii/S1557308 $71300142 \mathrm{X}$ 